\title{
THE RUN OF SUPERADIABATICITY IN STELLAR CONVECTION ZONES. II. EFFECT OF PHOTOSPHERIC CONVECTION ON SOLAR $p$-MODE FREQUENCIES
}

\author{
PIERRE DEMARQUe \\ Center for Solar and Space Research, Department of Astronomy, Yale University, New Haven, CT 06520-8101 \\ D. B. GUENTHER \\ Department of Astronomy and Physics, Saint Mary's University, Halifax, Nova Scotia B3A 4R2, Canada \\ AND \\ YONG-CHEOL KIM ${ }^{1}$ \\ Center for Space Astrophysics, Yonsei University, Seodaemoon-gu, Shinchon-dong 134, Seoul 120-749, Korea \\ Received 1998 April 7; accepted 1998 December 29
}

\begin{abstract}
Kim and Chan have completed a three-dimensional numerical simulation of the interaction between turbulent convection and radiation in and above the highly superadiabatic layer (SAL) in the Sun. They have shown that the dynamics of the domain dictates a SAL structure different from that of a traditional hydrostatic solar model. The top boundary of the convection zone is moved outward by about 0.3 pressure scale height, and in addition, convective overshoot extends into the radiative atmosphere layers by 0.45 pressure scale height. Using our one-dimensional stellar evolution code, we have studied the sensitivity of the calculated $p$-mode frequencies to a modification of the SAL structure similar to that predicted by Kim and Chan. We find that it is possible to remove in this way the main discrepancy between observed and calculated $p$-mode frequencies. This experiment is a promising first step in the use of physically realistic three-dimensional radiative-hydrodynamic numerical simulations to derive reliable surface boundary conditions for one-dimensional stellar models.
\end{abstract}

Subject heading: convection - Sun: interior - Sun: oscillations

\section{INTRODUCTION}

In the outer part of the solar envelope, there is a thin transition layer between the nearly adiabatic deep regions of the convection zone and the mostly radiative atmosphere. This transition layer is convectively unstable according to the Schwarzschild criterion. But because the density in this thin layer is very low, the efficiency of convective transport is also low. And as a consequence the layer is characterized by a temperature gradient that significantly exceeds the adiabatic temperature gradient. The structure of this highly superadiabatic layer (SAL) depends on the detailed interplay between the radiative and convective transports, and it is poorly understood.

The depth of the convective envelope and hence the radius of the Sun is determined by the specific entropy $S$ in the convection zone (Schwarzschild 1958; Larson 1974). Except for the SAL, most of the convection zone is adiabatically stratified; hence the specific entropy of the convection zone is determined predominately by the SAL. The structure of the SAL in turn depends on the opacities, equation of state, surface boundary conditions, and the model of convective energy transport. The model of convection used in nearly all stellar evolutionary calculations is based on the mixing-length approximation (MLA; Böhm-Vitense 1958). In the MLA the convective energy is assumed to be carried by an element of fluid of fixed size that rises a specific distance (the mixing length) and then is instantly absorbed by its surroundings through radiative diffusion. In stellar structure calculations the mixing-length distance is assumed to be proportional to the pressure scale height; the constant of proportionality, $\alpha$, is an adjustable parameter of the MLA.

\footnotetext{
${ }^{1}$ Work started at Center for Solar and Space Research, Department of Astronomy, Yale University, New Haven, CT 06520-8101.
}

In solar models, $\alpha$ is adjusted to produce a model with the correct radius. Regardless of this fine tuning, because the MLA is a crude approximation of the actual physics of convection, the predicted run of stellar structure variables in the convection zone and especially in the SAL, where the assumptions of the MLA break down, are unlikely to be correct (Guenther et al. 1992).

Helioseismology provides an opportunity to probe directly and sensitively the solar interior. The discrepancy between the computed and the observed frequencies of $p$-modes can be used to determine which parts of the solar model need further refinement. Indeed, the frequency dependence of the discrepancy reveals that there is a problem with the model and that the problem lies very near the surface. Specifically, solar models constructed with the MLA do not accurately represent the structure of the photospheric and subphotospheric layers near the SAL (Guenther 1994).

The need to develop a better model of convection for stellar evolutionary calculations goes beyond trying to match solar models with $p$-mode oscillations. We are motivated to understand better the atmospheric and SAL structure in order to gain the ability to calculate radii of cool stars from first principles and without the uncertainties associated with the MLA. In addition, understanding the energetics of the SAL will lead to a better description of convective overshoot into the atmosphere above the convection zone and possibly of overshoot or penetration into the radiatively stable interior below (Zahn 1991). The SAL has also been suggested as the site of the driving of the p-mode oscillations in stars (Kumar \& Goldreich 1989; Goldreich, Murray, \& Kumar 1994; Goode et al. 1998).

Recently, Kim \& Chan (1998) have completed a threedimensional radiative hydrodynamic simulation of the complete extent of the SAL including the solar atmosphere. 
Our eventual research goal is the implementation of a threedimensional numerical simulation like the Kim-Chan simulation into our stellar evolution code to serve as surface boundary condition of a model of the solar interior. That work is now in progress.

The purpose of this paper is to explore in a preliminary way the sensitivity of the $p$-mode frequencies to the kind of temperature gradient profile predicted in the atmosphere and near the SAL by the more realistic Kim \& Chan (1998) simulation. In $\S 2$ we summarize recent relevant research on the structure of the SAL in the Sun; $\S 3$ describes the main results of the Kim-Chan paper, i.e., the outward shift of the convective boundary and the effect of the hydrodynamics on the structure of the outer radiative layers. The KimChan work shows that the main issue is not the detailed treatment of convection in the deep layers, but rather how hydrodynamic motions affect the temperature stratification in the deep atmosphere and how this in turn affects the top of the convective envelope boundary. Our calibrated solar models are described in $\S 4$. They show that the findings of Kim \& Chan (1998) change the $p$-mode frequencies of calibrated solar models in the direction of improving agreement with observation. Finally, we summarize our results in $\S 5$.

\section{SOME RECENT STUDIES}

One way to improve the solar model and its description of the SAL is to introduce a theoretical model of turbulent convection into the construction of the one-dimensional stellar model that is physically more realistic than the MLA. Several approaches, either semianalytical or based on numerical simulations of convection, have been discussed recently in the context of solar modeling:

1. Canuto $(1990,1996)$ has proposed a semianalytical approach to this problem. Applications to stellar models have been discussed by Canuto \& Mazzitelli $(1991,1992)$ and Canuto, Goldman, \& Mazzitelli (1996). The main idea is to include a full turbulent spectrum in the model of convection. The parameters of the Canuto-Mazzitelli (C\&M) approach are derived from laboratory experiments of incompressible convection extrapolated to stellar conditions. In their approach, radiative transport is decoupled from convective transport and is treated as a diffusive process, as in the MLA.

The C\&M approach still requires a characteristic "mixing" scale. One can adopt a traditional mixing length that is proportional to the pressure scale height $H_{p}$ or, as Canuto and Mazzitelli prescribe, a length scale that at each point is set equal to the distance to the surface convection boundary $z$. In both cases, a scaling factor $\alpha$ is required to produce solar models with the correct radius. Stothers \& Chin (1995) have derived a convenient formulation of the C\&M theory, which is suitable for stellar structure calculations. When applied to the Sun, the Stothers-Chin formulation leads to a narrower superadiabatic peak than in the standard mixing-length case. This corresponds to a relatively lower convective efficiency just below the radiative/ convective boundary, since lower convective efficiency requires steeper temperature gradients to carry the same energy flux.

In Figure 1 we show the superadiabaticity $\left(\nabla-\nabla_{a d}\right)$, where $\nabla$ is the temperature gradient and $\nabla_{\text {ad }}$ is the adiabatic temperature gradient, near the surface for several solar models. The two models constructed using the C\&M

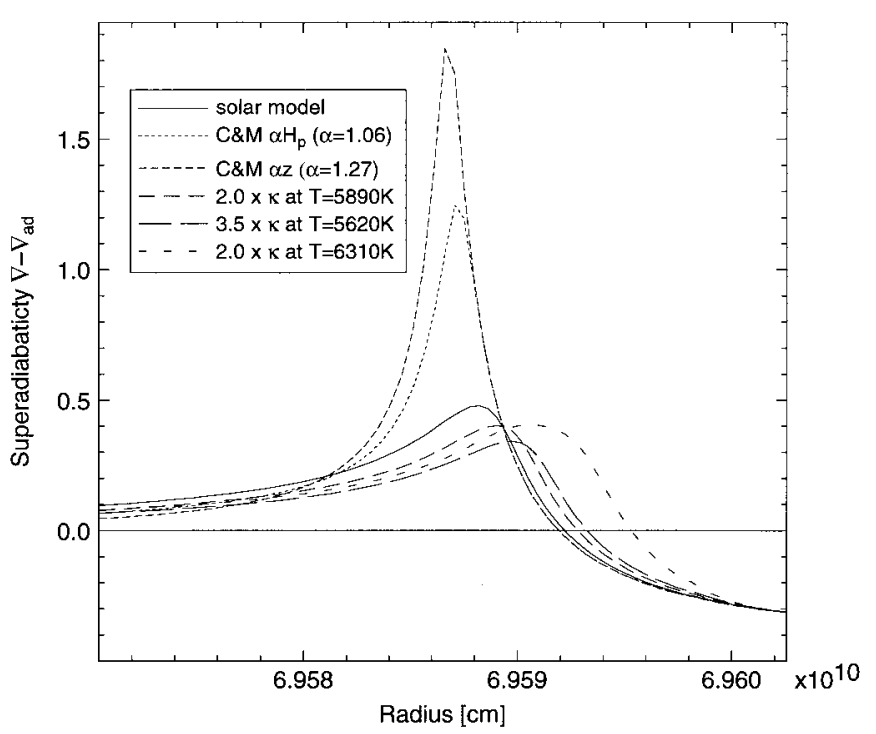

FIG. 1.- Structure of the highly superadiabatic layer in a reference standard MLA solar model (continuous line), in two C\&M models, and in the three perturbed- $\kappa$ models described in the text.

approach, one assuming that the mixing length is proportional to the pressure scale height and the other assuming that the mixing length is proportional to the depth, have the highest peaks. The superadiabaticity predicted by the $\mathrm{C} \& \mathrm{M}$ approach clearly differs from that predicted by the solar model, which is based on the conventional MLA.

Looking at the plot of the $p$-mode frequency differences (model minus observation) shown in Figure 2, one could argue that some of the C\&M models improve the agreement between the calculated $p$-mode frequencies and the $p$-mode frequencies observed on the Sun (Paterno et al. 1993). The solar models in Figure 2 were all calculated using the Yale Stellar Evolution Code (Guenther et al. 1992). The models include the latest physical treatments of equation of state, opacities, and nuclear energy generation. They also include gravitational settling of helium and heavy elements (see Guenther \& Demarque 1997 for a detailed description of the model physics). The theoretical $p$-modes were all calculated using Guenther's nonadiabatic stellar pulsation code (Guenther 1994). The observational data are from the Global Oscillation Network Group instrument (Harvey et al. 1996). Except for the model identified as "solar model (adiabatic)," all the theoretical $p$-mode frequencies include nonadiabatic corrections due to radiation (in the Eddington approximation). Note that it was necessary to scale the depth dependent mixing length in the C\&M models by a factor of 1.27 or to use a mixing length of $1.06 H_{p}$ in order to obtain a model with the observed solar radius.

2. Chan \& Sofia (1989) have performed a threedimensional numerical simulation of deep convection (i.e., in the regime where the superadiabatic excess in the temperature gradient is very small). They showed that in the deep regime the MLA is valid. They derived expressions for key physical parameters that, in principle, can be conveniently applied to stellar models, such as the convective energy flux.

The Chan-Sofia formula for the convective flux was incorporated into the Yale stellar evolution code by Lydon (1993) and Lydon, Fox, \& Sofia (1992). The diffusion approximation was used to treat radiative transfer, and the 


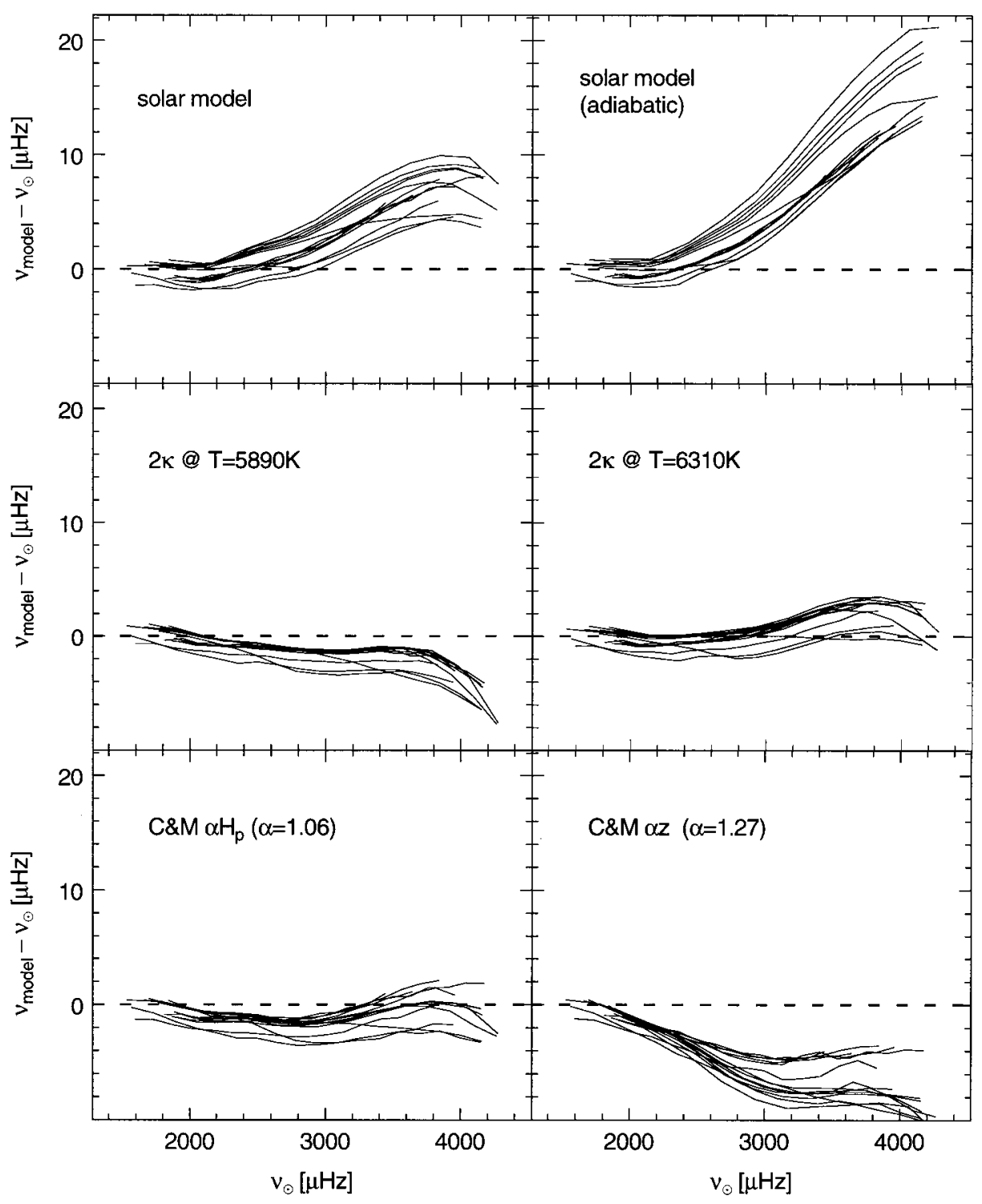

FIG. 2. $-P$-mode frequency difference diagrams, model minus observation, for several solar models. Common $l$-valued $p$-modes are connected with a line. Plotted are the $l=0,4,10,20,30,40,50,60,70,80$, and $100 p$-modes. Note the improvement in the calculated frequencies of the perturbed- $\kappa$ model labeled " $2 \kappa$ at $T=6310 \mathrm{~K}$ " in comparison with frequencies of the standard solar model shown in the top left-hand panel. See text for a discussion of the other models.

radiative atmosphere was treated by Lydon et al. (1992) as in standard MLA Yale models (e.g., Guenther et al. 1992). They found an improvement over the mixing-length formalism in the description of the velocity field below the SAL. They also found the peak of the SAL is not as high as in the C\&M model and is located at a greater depth below the surface. Their models yielded a larger discrepancy with the observed solar $p$-mode frequencies than in the equivalent MLA models. Part of the discrepancy was due to the fact that these models did not match the solar radius very precisely. But as pointed out by Lydon (1993) himself, extrapolating the convective flux approximation into the SAL, where the temperature gradient greatly exceeds the adiabatic gradient, is probably invalid.

3. In order to alleviate these difficulties, Kim (1993) and Kim et al. $(1995,1996)$ conducted a three-dimensional numerical simulation whose domain includes more shallow layers of the Sun and that treats the coupling of radiation and convection in the simulation itself. This simulation includes realistic physics for the equation of state and radiative opacities, taken from the Yale Stellar Evolution Code. The Kim et al. $(1995,1996)$ simulation confirms the result of Chan \& Sofia (1989) that in the deep adiabatic layers, the energy transport efficiency and temperature gradients are as predicted by the MLA. But it departs markedly from the MLA prediction in the shallow regions and the SAL, where partial ionization takes place and the importance of the radiative flux grows more rapidly than predicted by the MLA. This last conclusion was also reached by Canuto (1990, 1996).

A simple parameterization of the Kim et al. (1996) models was used by Demarque, Guenther, \& Kim (1997; hereafter Paper I) in precisely calibrated solar models. These models exhibit, like the C\&M models, a higher SAL peak maximum than the MLA models (see Fig. 1 of Paper I; see also Fig. 1 of Demarque, Guenther, \& Kim 1996). The $p$-mode frequencies of the models were found to agree more closely with the observed solar $p$-mode frequencies than the 
MLA model frequencies. The Kim et al. $(1995,1996)$ simulation treats radiative transfer in the diffusion approximation (optically thick medium) and as a consequence cannot be used in the solar atmosphere. Thus the top boundary of the simulation was set below the SAL peak.

Although encouraging, these results must be interpreted with some caution for the following reasons:

1. All the above models include an oversimplified description of the atmospheric layers of the Sun. The outer atmospheres are assumed to be radiative until, moving inward, convection sets in according to the Schwarzschild criterion. In particular, there is no provision for convective overshoot (from the top of the convection zone into the base of the atmosphere), which is known to take place and could modify the structure and affect the $p$-mode frequencies.

2. Even though some of the models described above yield calculated $p$-mode frequencies in better agreement with observation than standard MLA models, one must emphasize that this does not prove the physics of the models is more correct. Similar structural modifications can be obtained from a variety of alterations to the model physics. Because of the nonlinearity of the problem and the relatively low sensitivity to the outermost layers, there is no unique model that improves the $p$-mode frequencies using the low- and intermediate- $l$ values that are now available (see the discussion by Guenther, Kim, \& Demarque 1996 and Figs. 2 and 3 in Demarque et al. 1996). But note that each model predicts a markedly different structure for the atmospheric layers, and it is expected that future observations of high-l modes, which are mostly sensitive to the superficial layers, will provide the needed discriminant between models.

Guenther $(1991,1994)$ has explored the importance of the treatment of radiative transfer in the surface layers. It is also in this region that nonadiabatic effects are the most significant. The nonadiabatic pulsation code of Guenther (1994) used in this study evaluates the radiative gains and losses using the Eddington approximation, which is a definite improvement compared to the diffusion approximation. The diffusion approximation, although useful in the deep layers, is invalid in the optically thin regions.

\section{ROLE OF CONVECTION AND CONVECTIVE OVERSHOOT IN THE ATMOSPHERE}

As mentioned above, the meaning of the improved agreement in the $p$-mode frequencies based on the models of $\S 2$ can be questioned. To respond to these doubts requires a more realistic treatment of the outer layers, including a description of convective overshoot from a radiativehydrodynamic treatment of the solar atmosphere. One approach, used in the context of inversions of the solar $p$-mode frequencies, has been to introduce turbulent pressure as a free parameter in the hydrostatic equilibrium equations (Balmforth 1992a, 1992b, 1992c; Rosenthal 1997; Antia \& Basu 1997). The justification is that the dynamical nature of convective motions is responsible for a turbulent pressure $P_{\text {turb }}$, which must now be added to the gas and radiation pressures, so that the local total pressure is the sum of the gas plus radiation plus turbulent pressures. A simple estimate of the ratio of turbulent to gas pressure in the SAL shows that this ratio could reach values as high as 0.5 in the subsonic case, depending on the turbulent velocity field $v_{\text {turb }}$ (Cox \& Giuli 1968). Thus $P_{\text {turb }}$ can be used as an adjustable parameter in inversions of the observed $p$-mode oscillation frequencies. Similarly, macroturbulence has traditionally been incorporated in model atmospheres to account for the observed broadening of absorption lines (Mihalas 1978). Since turbulent convection is a threedimensional phenomenon and solar models are onedimensional, $P_{\text {turb }}$ is a convenient parameter to use. It is possible to produce a model that matches the observed $p$-mode frequencies more closely by adjusting $P_{\text {turb }}$. Here $P_{\text {turb }}$ plays a role similar to the mixing-length parameter used in the MLA to match the observed solar radius. But in fact the local value of $P_{\text {turb }}$ is not a free parameter, and it should in principle be derived from the local physics in a realistic convection simulation.

Schlattl, Weiss, \& Ludwig (1997) have successfully incorporated the results of a two-dimensional simulation of radiative hydrodynamics by Freytag, Ludwig, \& Steffan (1996) in a solar model. The Freytag et al. (1996) simulation shows that the atmospheric structure is sensitive to hydrodynamic effects. Unfortunately, it does not include the full extent of the solar SAL. Schlattl et al. (1997) have approximated the structure of the deeper layers in their solar models and found an improvement on the calculated $p$-mode frequencies.

More recently, Kim \& Chan (1998) have completed a three-dimensional radiative hydrodynamic simulation of the complete extent of the SAL including the solar atmosphere (about 2 pressure scale heights above and 2.5 pressure scale heights below the SAL). The numerical approach was described by Kim \& Chan (1997). A similar domain was simulated by Nordlund and his collaborators (Nordlund \& Dravins 1990; Nordlund \& Stein 1997; see also the review by Chan et al. 1991) using different numerical methods for the convective and radiative components. More recently, Abbett et al. (1997) have also discussed the same transition layer. The simulation of Kim \& Chan (1998) is fully compressible and uses realistic microscopic physics. The radiation has been treated utilizing the three-dimensional Eddington approximation, which is valid in the optically thin regions near the surface. We alert the reader that the solar model from which the three-dimensional simulation was initiated was generated in 1994, and as a result differs from our current solar models in two significant ways: (1) the solar model does not include the effects of gravitational settling of helium or heavier elements, and (2) the solar model does not use the latest OPAL (Opacities Livermore) equation of state tables.

In this paper, we use the term overshoot in the sense proposed by Zahn (1991): i.e., to describe layers beyond the formal convective boundary in which motions take place, in some cases leading to composition mixing, but where the mean temperature gradient is subadiabatic, in contrast with convective penetration in which superadiabatic convective cells travel into the radiative layers. From the point of view of this paper, the Kim \& Chan (1998) paper contains two significant new results:

1. The three-dimensional numerical simulation exhibits convective instability in layers which were radiative in the hydrostatic used as initial model. This outward shift of the convective boundary can be seen in Figure $3 a$. The point where $\nabla-\nabla_{\text {ad }}$ drops below zero for the three-dimensional simulation, which according to the Schwarzschild criterion marks the transition from convective to radiative stability, 
is shifted outward by about $0.3 H_{p}$ compared to the initial model. Furthermore, the superadiabatic peak is shifted outward by $\approx 0.15 H_{p}$, fattened, and diminished in height. This is exactly the opposite of the effect predicted by Canuto \& Mazzitelli (1992; Fig. 1) and by our earlier simulations extrapolated into the SAL (Paper I).

2. In addition, the three-dimensional numerical simulation exhibits convective overshooting by about $0.45 H_{p}$ into the radiative layers of the atmosphere. Note that in the region of overshoot, the mean temperature gradient is subadiabatic and that it cannot therefore be obviously distinguished in Figure $3 a$ from the mean temperature gradient in the more superficial radiative layers. However, turbulent pressure plays a role in the structure of the overshoot region, affecting the mean stratification of the atmosphere and modifying the position of the onset of convection.

The next logical step would be to incorporate the Kim \& Chan (1998) simulation into a model of the Sun. The $p$-mode frequencies calculated from the resulting improved solar model would provide an excellent test of the validity of numerical simulations of convection and of their ability to calculate the run of the temperature gradient sufficiently well to reproduce the solar radius. This challenging task is now in progress.

Before this is achieved, we explore here in a preliminary way the sensitivity of the $p$-mode frequencies to the kind of temperature gradient profile predicted in the atmosphere and near the SAL by the more realistic Kim \& Chan (1998)

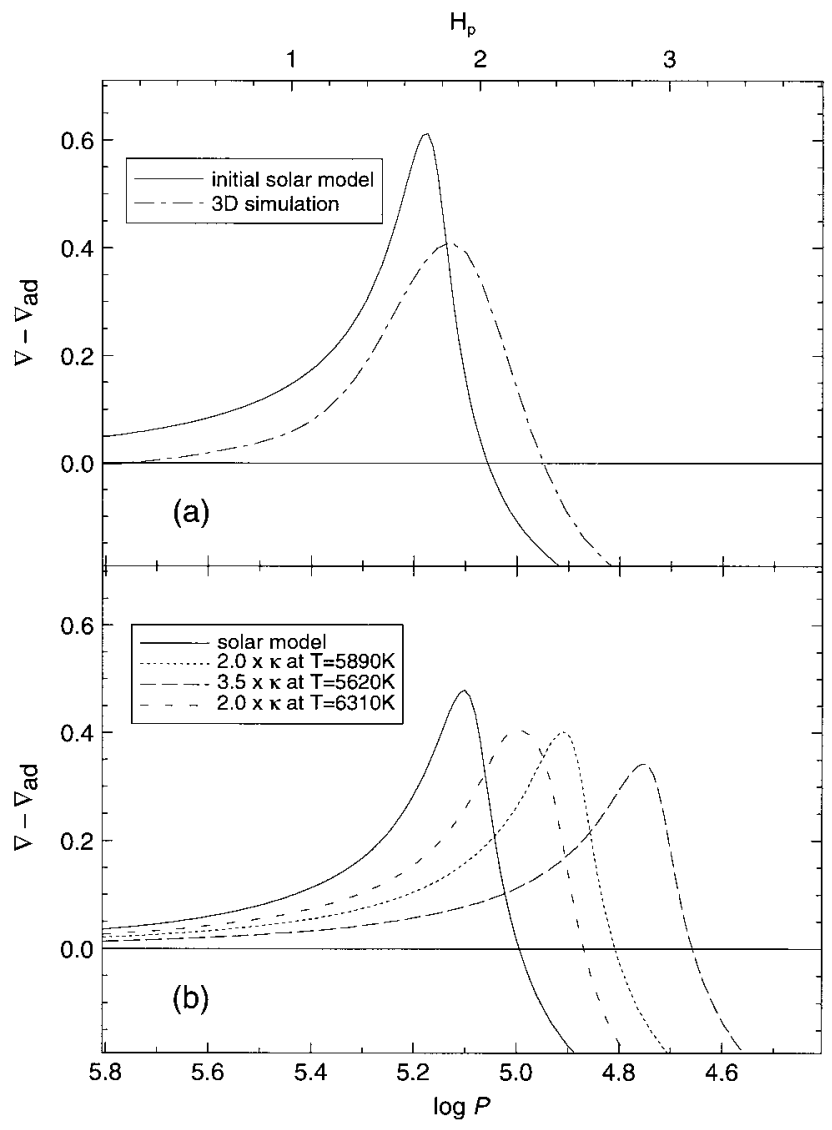

FIG. 3.-(a) SAL structure as a function of log-pressure (lower scale) and pressure scale height $H_{p}$ (upper scale) for the initial solar model used in the Kim \& Chan (1998) simulation. Note that the initial solar model shown in this figure is of older generation and is slightly different from the reference solar model plotted in the figure below. (b) Same as above for the reference solar model and for the three perturbed- $\kappa$ models. simulation. Within the context of a one-dimensional solar model, the presence of turbulence in the radiative layers at the top of the convection zone steepens the temperature gradient and effectively moves the boundary of convective instability at the surface outward. Introducing an artificial bump in the opacity in the vicinity of the convective boundary can simulate this effect on the temperature gradient (Guenther et al. 1996) in the one-dimensional model. Such models are illustrated in Figure 1. A similar approach has previously been adopted by Guzik \& Cox (1995), who were able to match the observed solar $p$-mode frequencies very faithfully.

\section{CALIBRATED SOLAR MODELS}

For the purpose of comparison, a modern reference standard solar model was constructed with the Yale Stellar Evolution Code. The OPAL opacities tables (Iglesias \& Rogers 1996) were used together with the low-temperature opacities from Kurucz (1991). The equation of state was taken from Rogers, Swenson, \& Iglesias (1996). When out of the table, the Yale standard implementation with the Debye-Hückel correction was used (Guenther et al. 1992). Helium and heavy element diffusion processes were included in the models. In this case, gravitational settling is the dominant diffusion process, and selective radiation processes can safely be ignored. Heavy element diffusion was implemented by assuming that all heavy elements diffuse with the same velocity as fully ionized iron (Guenther \& Demarque 1997). Note that all models are evolved from the zero-age main sequence to the current solar age. The mixing-length ratio and the helium content $Y$ have been adjusted in the usual way so as to match the solar luminosity, radius, and the observed solar ratio of heavy element to hydrogen abundances $Z / X=0.0245$ (Grevesse \& Noels 1993; Grevesse, Noels, \& Sauval 1996).

In order to mimic the SAL structure of the Kim \& Chan (1998) simulation, shown in Figure $3 a$ (namely, to shift the top of the convection zone to a shallower layer), we have increased the surface opacity in the way described by Guenther et al. (1996). We refer to these models as perturbed- $\kappa$ solar models. The amount, location, and extent of the perturbation were systematically varied to study the effect on solar $p$-mode. We note that this procedure is equivalent to introducing an ad hoc turbulent pressure term in the region where the opacity is enhanced (Demarque, Guenther, \& Kim 1998).

Three perturbed- $\kappa$ models are displayed in Figure $3 b$, where superadiabaticity is plotted versus $\log _{10} P$. All three models are constructed with the atmospheric opacity convolved with a bell-shaped weight function curve in the $\log _{10}$ $T$ domain. The width of the bell curve is \pm 0.5 in $\log _{10} T$, the maximum multiplication factor, and the locations are 2 at $\log _{10} T=3.77,3.5$ at $\log _{10} T=3.75$, and 2 at $\log _{10}$ $T=3.80$.

Figure $3 b$ illustrates the corresponding shifts in the top convective boundary in the perturbed- $\kappa$ models. Expressed in terms of the pressure scale height $H_{p}$, these shifts are approximately $0.9 H_{p}, 0.5 H_{p}$, and $0.33 H_{p}$, as compared to the standard solar model. The shift of $0.33 H_{p}$ in the third perturbed- $\kappa$ model (a maximum multiplication factor of 2 at $\log _{10} T=3.80$ ) is close to that of the Kim \& Chan (1998) simulation.

Figure 2 illustrates the sensitivity of the frequency difference diagram slope to the position of the onset of convec- 
tion. As the convective boundary is moved out into the atmosphere (while the radii of the models remain tuned to the observed solar radius to one part in $10^{6}$ ), the discrepancy between calculated and observed $p$-mode frequencies decreases when compared to the reference solar model and then increases with the opposite slope. We see in Figure 2 that the opacity tweak that shifts the superadiabatic peak by $0.33 H_{p}$ (at $T=6310 \mathrm{~K}$ ) produces $p$-modes in very close agreement with observation. On the other hand, when the same opacity tweak is applied at $\log _{10} T=3.77$ (i.e., $T=5890 \mathrm{~K}$ ), the frequency differences increase in magnitude at the high-frequency end and change sign. Thus, although our models provide only an approximate representation of the mean temperature gradient in the Kim \& Chan (1998) simulation, they confirm the importance of convection and overshoot in the deep atmospheric layers.

\section{SUMMARY}

The low- and intermediate- $l p$-modes do make it clear that the structure near the surface of the MLA-based solar model is not completely accurate. A recent threedimensional radiative hydrodynamic numerical simulation by Kim \& Chan (1998), whose domain covers the complete extent of the SAL, including the solar atmosphere (about 2 pressure scale heights above and 2.5 pressure scale heights below the SAL), indicates that the top boundary of the convection zone should be moved outward by about 0.3 pressure scale height. It is curious that the sharply peaked C\&M-based solar model and the broader peaked KimChan-based models can both have a positive effect on the $p$-mode spectrum. For the low- and intermediate-l $p$-modes examined, the radial wavelengths of the $p$-modes cannot resolve the structural differences that exist between these models in the thin SAL. Higher order $p$-modes will be required to resolve the differences. Because the physics of the numerical three-dimensional convection based models is more realistic and complete, we favor at this time the results of these simulations over models based on the MLA and the $C \& M$ formulation.

We have studied the sensitivity of the $p$-mode frequencies to a shallower location of the top boundary of convection, as predicted by the numerical simulations. We conclude that the top of the convection zone is located too deeply in our standard solar model.

The next objective in this research will be to incorporate the structure of the outer layers of the Sun derived from a physically realistic three-dimensional simulation, such as the Kim \& Chan (1998) simulation, into a solar interior model in a way that is consistent with the interior physics of the Sun and that strictly satisfies all the constraints of a precisely calibrated standard solar model. The tests we have carried out suggest that such a model would bring the calculated $p$-modes to within a few $\mu \mathrm{Hz}$ of the observed $p$ modes. It must be emphasized here that, because of the many simplifying assumptions in the best available models (in particular the neglect of atmospheric inhomogeneities and motions), more precise agreement with all observed $p$-mode frequencies should not be expected. But it seems that it is now within the reach of present numerical simulations of radiative hydrodynamics to provide surface boundary conditions that will yield the correct solar radius without any recourse to the adjustable parameter $\alpha$, a longawaited advance in the theory of stellar evolution.

This work was supported in part by NASA grant NAG52795 (P. D. and Y. C. K.), an operating grant (to D. B. G.) from the National Science and Engineering Research Council of Canada, and by the Creative Research Initiative Program of the Korean Ministry of Science and Technology (Y. C. K.). We are indebted to an anonymous referee whose constructive critical comments greatly improved this paper.

\section{REFERENCES}

Abbett, W. P., Beaver, M., Davids, B., Georgobiani, D., Rathbun, B., \& Stein, R. F. 1997, ApJ, 480, 395

Antia, H. M., \& Basu, S. 1997, Ap\&SS, 225, 51

Balmforth, N. 1992a, MNRAS, 255, 603

. 1992b, MNRAS, 255, 632 1992c, MNRAS, 255, 639

Böhm-Vitense, E. 1958, Z. Astrophys., 46, 108

Canuto, V. M. 1990, A\&A, 227, 282 1996, ApJ, 467, 385

Canuto, V. M., Goldman, I., \& Mazzitelli, I. 1996, ApJ, 473, 550

Canuto, V. M., \& Mazzitelli, I. 1991, ApJ, 370, 275 1992, ApJ, 389, 724

Chan, K. L., Nordlund, A., Steffen, M., \& Stein, R. F. 1991, Solar Interior \& Atmosphere, ed. A. N. Cox, W. C. Livingston, \& M. S. Matthews (Tucson: Univ. Arizona Press), 223

Chan, K. L., \& Sofia, S. 1989, ApJ, 336, 1022

Cox, J. P., \& Giuli, R. T. 1968, Principles of Stellar Structure (New York: Gordon and Breach)

Demarque, P., Guenther, D. B., \& Kim, Y.-C. 1996, in Proc. 32d Liège Astrophys. Colloq., Stellar Evolution: What Should Be Done?, ed. A. Noels, D. Fraipont-Caro, M. Gabriel, N. Grevesse, \& P. Demarque (Liège: Univ. Liège), 279 1997, ApJ, 474, 790

1998 in Structure and Dynamics of the Interior of the Sun and Sun-like Stars, ed. S. G. Korzennik \& A. Wilson (ESA SP-418; Noordwijk: ESA)

Freytag, B., Ludwig, H.-G., \& Steffen, M. 1996, A\&A, 313, 497

Goldreich, P., Murray, N., \& Kumar, P. 1994, ApJ, 424, 466

Goode, P. R., Strous, L. H., Rimmele, T. R., \& Stebbins, R. T. 1998, ApJ, 495, L27

Grevesse, N., \& Noels, A. 1993, in Origin and Evolution of the Elements, ed. N. Prantzos, E. Vangioni-Flam, \& M. Cassé (Cambridge: Cambridge Univ. Press), 15

Grevesse, N., Noels, A., \& Sauval, A. J. 1996, in ASP Conf. Ser. 99, Cosmic Abundances, ed. S. S. Holt \& G. Sonneborn (San Francisco: ASP), 117
Guenther, D. B. 1991, ApJ, 369, 247 1994, ApJ, 422,400

Guenther, D. B., \& Demarque, P. 1997, ApJ, 484, 937

Guenther, D. B., Demarque, P., Kim, Y.-C., \& Pinsonneault, M. H. 1992, ApJ, 387, 372

Guenther, D. B., Kim, Y.-C., \& Demarque, P. 1996, ApJ, 463, 382

Guzik, J. A., \& Cox, A. N. 1995, ApJ, 448, 905

Harvey, J. W., et al. 1996, Science, 272, 1284

Iglesias, C. A., \& Rogers, F. J. 1996, ApJ, 464, 943

Kim, Y.-C. 1993, Ph.D. thesis, Yale Univ.

Kim, Y.-C., \& Chan, K. L. 1997, Ap\&SS, 225, 131 1998, ApJ, 496, L121

Kim, Y.-C., Fox, P. A., Demarque, P., \& Sofia, S. 1996, ApJ, 461, 499

Kim, Y.-C., Fox, P. A., Sofia, S., \& Demarque, P. 1995, ApJ, 442, 422

Kumar, P., \& Goldreich, P. 1989, ApJ, 342, 558

Kurucz, R. L. 1991, in Stellar Atmospheres: Beyond Classical Models, ed.

L. Crivellari, I. Hubeny, \& D. G. Hummer (Dordrecht: Kluwer), 440

Larson, R. B. 1974, Fundam. Cosmic Phys., 1, 1

Lydon, T. J. 1993, Ph.D. thesis, Yale Univ.

Lydon, T. J., Fox, P. A., \& Sofia, S. 1992, ApJ, 397, 701

Mihalas, D. 1978, Stellar Atmospheres (2d ed.; San Francisco: W. H. Freeman and Company)

Nordlund, Å., \& Dravins, D. 1990, A\&A, 228, 155

Nordlund, A., \& Stein, R. F. 1997, Ap\&SS, 225, 79

Paterno, L., Ventura, L., Canuto, V., \& Mazzitelli, I. 1993, ApJ, 402, 733

Rogers, F. J., Swenson, F. J., \& Iglesias, C. A. 1996, ApJ, 456, 902

Rosenthal, C. S. 1997, Ap\&SS, 225, 145

Schlattl, H., Weiss, A., \& Ludwig, H.-G. 1997, A\&A, 322, 646

Schwarzschild, M. 1958, Structure and Evolution of the Stars (Princeton: Princeton Univ. Press)

Stothers, R. B., \& Chin, C.-W. 1995, ApJ, 440, 297

Zahn, J.-P. 1991, A\&A, 252, 179 